\title{
Distributionally Robust Return-Risk Optimization Models and Their Applications
}

\author{
Li Yang, ${ }^{1}$ Yanxi Li, ${ }^{1}$ Zhengyong Zhou, ${ }^{2}$ and Kejing Chen ${ }^{1}$ \\ ${ }^{1}$ Faculty of Management and Economics, Dalian University of Technology, Dalian 116024, China \\ ${ }^{2}$ School of Mathematics and Computer Sciences, Shanxi Normal University, Linfen 041004, China \\ Correspondence should be addressed to Li Yang; yangli96@aliyun.com
}

Received 27 February 2014; Accepted 5 May 2014; Published 20 May 2014

Academic Editor: Ying $\mathrm{Hu}$

Copyright (c) 2014 Li Yang et al. This is an open access article distributed under the Creative Commons Attribution License, which permits unrestricted use, distribution, and reproduction in any medium, provided the original work is properly cited.

\begin{abstract}
Based on the risk control of conditional value-at-risk, distributionally robust return-risk optimization models with box constraints of random vector are proposed. They describe uncertainty in both the distribution form and moments (mean and covariance matrix of random vector). It is difficult to solve them directly. Using the conic duality theory and the minimax theorem, the models are reformulated as semidefinite programming problems, which can be solved by interior point algorithms in polynomial time. An important theoretical basis is therefore provided for applications of the models. Moreover, an application of the models to a practical example of portfolio selection is considered, and the example is evaluated using a historical data set of four stocks. Numerical results show that proposed methods are robust and the investment strategy is safe.
\end{abstract}

\section{Introduction}

The mean-variance portfolio model developed by Markowitz [1] is considered as the beginning of the financial investment research. The return and risk of a portfolio were measured by the expected value and the variance of the random portfolio return in this model. The risk measures "value-at-risk" (VaR) and "conditional VaR" (CVaR) have been studied extensively in recent literature of risk management and portfolio selection. However, when return distributions are not elliptical, the variance and VaR fail for risk evaluations. Artzner et al. [2], Mausser and Rosen [3], Rockafellar and Uryasev [4], and Uryasev and Rockafellar [5] also showed that CVaR has the most desirable properties. For example, it satisfies subadditivity property and looks deeply into the tail of a distribution. To cope with nonnormal elliptical distributions, $\mathrm{CVaR}$ may be compared with the widely accepted VaR risk performance measure. Based on the risk control of CVaR, Lin and Gong [6] established a mean-CVaR model on the basis of the mean-variance model and under normal distribution condition. Assuming that security rates of return have a multivariate normal distribution, Alexander and Baptista [7] analyzed the portfolio selection implications arising from imposing a VaR or a CVaR constraint on the mean-variance model. They showed that a CVaR constraint dominates a $\mathrm{VaR}$ constraint as a risk management tool when a risk-free security is present. Based on CVaR risk measure, Andersson et al. [8] examined a new approach for credit risk optimization. In this approach, the credit risk distribution was generated by Monte Carlo simulations, so it was not necessary to assume that the credit risk distribution is known. The optimization problem was solved by linear programming. However, generated several thousands of scenarios may lead to the linear programming with huge number of variables and constraints thus decreasing the computational efficiency of the problem. To overcome this difficulty, nondifferential optimization techniques and linear programming duality theory were explored in [9] and [10], respectively.

Robust optimization refers to finding a strategy whose behavior under the worst possible realizations of the uncertain inputs is optimized, to deal with uncertain data in optimization models. Robust optimization models have been designed and discussed extensively; see, for example, BenTal et al. [11] and Bertsimas et al. [12] and the references therein. The research on robust optimization in both theory and application was surveyed in [12]. Ogryczak [13] showed 
that for box uncertainty the robust model of the mean is essentially a CVaR, and also the robust model of the CVaR itself is a CVaR with appropriately redefined probabilities. Based on the worst-case CVaR, Gao and Bian [14] gave an objective model considering the overall investment period's risk level and derived a dynamic optimal portfolio model. Using vector autoregressive method and Monte Carlo simulation, the concrete steps for solving the optimal investment strategy were presented. Zhu and Fukushima [15] considered the worst-case CVaR in the situation where only partial information on the underlying probability distribution was available. The minimization of the worst-case CVaR under mixture distribution uncertainty, box uncertainty, and ellipsoidal uncertainty was investigated. Its application to robust portfolio optimization was given, and the corresponding problems were cast as linear programming and second-order cone programming. Tong and Liu [16] presented a worst-case $\mathrm{CVaR}$ index under the knowing part information of random variable, and three profit-risk robust portfolio models were proposed. The models were restated as linear programming problems for the case of box discrete distribution of random variable and the linear loss function. Bertsimas et al. [17] proposed a semidefinite optimization model for a class of minimax two-stage stochastic linear optimization problems with risk aversion. The distribution of second-stage random variables belongs to an uncertainty set of multivariate distributions with known first- and second-order moments. Zymler et al. [18] developed tractable semidefinite programming based approximations for distributionally robust individual and joint chance constraints, assuming that only the firstand second-order moments as well as the support of the uncertain parameters are given. They demonstrated that the worst-case $\mathrm{CVaR}$ can be computed efficiently for some classes of constraint functions. And the worst-case CVaR approximation for joint chance constraints was studied. Delage and Ye [19] proposed a model that describes uncertainty in both the distribution form and moments of random variable. Its application to portfolio selection that maximizes expected utility obtained from the single-step return of investment portfolio was given, and the problem was reformulated as a semidefinite programming problem when random variable belongs to $\mathbb{R}^{n}$ or an ellipsoidal set.

In this paper, based on the risk control of $\mathrm{CVaR}$, we propose distributionally robust return-risk optimization models, with box constraints of a random vector and the uncertainty in both the distribution form and moments of a random vector. The models are restated equivalently as semidefinite programming problems, which is computationally tractable both practically and theoretically. Moreover, an application of the models to a practical example of portfolio selection and corresponding numerical results are given.

The present paper is organized as follows. After this introduction in Section 2 distributionally robust return-risk optimization models are presented. Using the conic duality theory and the minimax theorem, the models are reformulated as semidefinite programming problems. In Section 3 we apply the models to a portfolio selection problem, and the problem is evaluated using a historical data set. Numerical results show that our proposed methods are robust and the obtained investment strategy is safe. Finally Section 4 is devoted to research perspective.

\section{Distributionally Robust Return-Risk Optimization Models}

In this section, we propose distributionally robust return-risk optimization models with first- and second-order moments uncertainty and box constraints of random vector. Their equivalent semidefinite programming formulations are presented.

Let $x \in \mathbb{R}^{n}$ be the decision variable, let $\xi \in \mathbb{R}^{m}$ be the random vector, and let $P(\xi)$ be the probability distribution of the random vector $\xi$. Suppose that a loss function is defined as $f(x, \xi)=-x^{T} \xi$. From [4], the CVaR performance function $\mathrm{CVaR}_{\beta}(x)$ can be written as

$$
\operatorname{CVaR}_{\beta}(x)=\min _{\alpha \in \mathbb{R}} F_{\beta}(x, \alpha),
$$

where $\beta \in \mathbb{R}$ is a confidence level,

$$
\begin{gathered}
F_{\beta}(x, \alpha)=\alpha+(1-\beta)^{-1} \int_{\xi \in \mathbb{R}^{m}}[f(x, \xi)-\alpha]^{+} d P(\xi), \\
{[f(x, \xi)-\alpha]^{+}=\max \{0, f(x, \xi)-\alpha\} .}
\end{gathered}
$$

In many economic and financial planning models, which depend on uncertain parameters (for example future interest rates, future demands for a product, or future commodity prices), their probability distribution is usually unknown. In such situations, using historical data of uncertain parameters, lower and upper bounds on the parameters and empirical estimates of the mean and covariance matrix of the parameters can usually be obtained. We use them to define a distributional uncertainty set below. Distributional set taking into account moments' uncertainty in random vector and box constraints of random vector is as follows:

$$
\begin{aligned}
& \mathscr{P}\left(\underline{\xi}, \bar{\xi}, \bar{\mu}, \bar{\Sigma}, \gamma_{1}, \gamma_{2}\right) \\
& =\left\{\begin{aligned}
& P(\xi \in S)=1, \quad S=\{\xi: \underline{\xi} \leq \xi \leq \bar{\xi}\} \\
P(\xi) \in M: & \left(\mathbb{E}_{\xi}[\xi]-\bar{\mu}\right)^{T} \bar{\Sigma}^{-1}\left(\mathbb{E}_{\xi}[\xi]-\bar{\mu}\right) \leq \gamma_{1} \\
& \mathbb{E}_{\xi}[\xi-\bar{\mu}]\left(\mathbb{E}_{\xi}[\xi-\bar{\mu}]\right)^{T} \leq \gamma_{2} \bar{\Sigma}
\end{aligned}\right\},
\end{aligned}
$$

where $M$ is the set of all probability measures on the measurable space $\left(\mathbb{R}^{m}, \mathfrak{B}\right), \mathfrak{B}$ is the Borel $\sigma$-algebra on $\mathbb{R}^{m}, \underline{\xi}$ and $\bar{\xi}$ are lower and upper bounds on the random vector $\xi, \underline{\xi}<\bar{\xi}, \bar{\mu}$ and $\bar{\Sigma}$ are estimates of the mean and covariance matrix of the random vector $\xi$, respectively, and the constants $\gamma_{1} \geq 0$ and $\gamma_{2} \geq 1$ quantify one's confidence in $\bar{\mu}$ and $\bar{\Sigma}$, respectively. Same as inequalities in [19], the second inequality in $\mathscr{P}(\cdot)$ assumes that the mean of $\xi$ lies in an ellipsoid of size $\gamma_{1}$ centered at the estimate $\bar{\mu}$, and the last inequality in $\mathscr{P}(\cdot)$ assumes that the "centered second-moment matrix" of $\xi$ lies in a positive semidefinite cone defined with a matrix inequality.

Based on the distributional set and classical portfolio optimization models, we set up the following distributionally robust return-risk optimization models. 
Model 1. Maximize the worst-case expected return with constraint including worst-case CVaR:

$$
\begin{array}{ll}
\max _{x} & \min _{P \in \mathscr{P}} \mathbb{E}_{\xi}[-f(x, \xi)] \\
\text { s.t. } & \max _{P \in \mathscr{P}} \min _{\alpha \in \mathbb{R}} F_{\beta}(x, \alpha) \leq \sigma_{1}, \\
& x \in \chi,
\end{array}
$$

where $\chi=\left\{x: \sum_{i=1}^{n} x_{i}=1, x \geq 0\right\} \subseteq \mathbb{R}^{n}$ is a convex set and the upper bound $\sigma_{1}>0$ on CVaR is given constant.

Model 2. Minimize the worst-case CVaR with constraint including the worst-case expected return:

$$
\begin{array}{ll}
\min _{x} & \max _{P \in \mathscr{P}} \min _{\alpha \in \mathbb{R}} F_{\beta}(x, \alpha) \\
\text { s.t. } & \min _{P \in \mathscr{P}} \mathbb{E}_{\xi}[-f(x, \xi)] \geq \sigma_{2}, \\
& x \in \chi,
\end{array}
$$

where the lower bound $\sigma_{2}>0$ on expected return is given constant.
Model 3. Maximize the objective function including worstcase expected return and CVaR:

$$
\max _{x \in \chi} \min _{P \in \mathscr{P}}\left(\mathbb{E}_{\xi}[-f(x, \xi)]-\sigma_{3} \min _{\alpha \in \mathbb{R}} F_{\beta}(x, \alpha)\right),
$$

where $\sigma_{3} \geq 0$ is a risk-aversion constant used to trade off the reward (expected return) and risk (CVaR).

Remark 1. The objective function of Model 3 represents a risk-adjusted expected return function. In the models above, $\sigma_{i}(i=1,2,3)$ should be given at first. Model 2 with bounded return is more common than Model 1 with bounded risk, because it is not so easy to expect an investor to specify maximum risk aversion levels with confidence. See, for example, Mansini et al. [20] and the references therein. The consideration to robust versions of the Sharpe ratio model [21] without requiring bounds on reward or risk will be included in our future work.

Because it is not easy to solve the models numerically, we give their tractable formulations by the following theorems. In the theorems, we use the following notation: the scalar product of any two symmetric matrices $A$ and $B$ is defined as $A \cdot B=\operatorname{Tr}\left(A^{T} B\right)$, where "Tr" denotes the trace (sum of diagonal elements) of a matrix.

Theorem 2. Model 1 can be reformulated as the following semidefinite programming problem:

$$
\begin{aligned}
& \max -\left(\gamma_{2}\left(\bar{\Sigma} \cdot Q_{1}\right)-\bar{\mu}^{T} Q_{1} \bar{\mu}+r_{1}+\bar{\Sigma} \cdot P_{1}-2 \bar{\mu}^{T} p_{1}+\gamma_{1} s_{1}\right) \\
& \text { s.t. } \quad\left(\begin{array}{cc}
r_{1}+\lambda_{1}^{T} \xi-\lambda_{2}^{T} \bar{\xi}-r_{3} & \frac{\left(q_{1}+x+\lambda_{2}-\lambda_{1}\right)^{T}}{2} \\
\frac{\left(q_{1}+x+\lambda_{2}-\lambda_{1}\right)}{2} & Q_{1}
\end{array}\right) \geq 0 \text {, } \\
& \left(\begin{array}{cc}
r_{2}+\lambda_{3}^{T} \underline{\xi}-\lambda_{4}^{T} \bar{\xi}-r_{4} & \frac{\left(q_{2}+\lambda_{4}-\lambda_{3}\right)^{T}}{2} \\
\frac{\left(q_{2}+\lambda_{4}-\lambda_{3}\right)}{2} & Q_{2}
\end{array}\right) \geq 0 \\
& \left(\begin{array}{cc}
r_{2}+\lambda_{5}^{T} \underline{\xi}-\lambda_{6}^{T} \bar{\xi}-r_{5} & \frac{\left(q_{2}+(x /(1-\beta))+\lambda_{6}-\lambda_{5}\right)^{T}}{2} \\
\frac{q_{2}+(x /(1-\beta))+\lambda_{6}-\lambda_{5}}{2} & Q_{2}
\end{array}\right) \geq 0, \\
& \left(\begin{array}{cc}
P_{1} & p_{1} \\
p_{1}^{T} & s_{1}
\end{array}\right) \geq 0, \quad\left(\begin{array}{cc}
P_{2} & p_{2} \\
p_{2}^{T} & s_{2}
\end{array}\right) \geq 0 \\
& \gamma_{2}\left(\bar{\Sigma} \cdot Q_{2}\right)-\bar{\mu}^{T} Q_{2} \bar{\mu}+r_{2}+\bar{\Sigma} \cdot P_{2}-2 \bar{\mu}^{T} p_{2}+\gamma_{1} s_{2} \leq \sigma_{1}, \\
& p_{1}=-\frac{q_{1}}{2}-Q_{1} \bar{\mu}, \quad p_{2}=-\frac{q_{2}}{2}-Q_{2} \bar{\mu}, \\
& r_{3} \geq 0, \quad r_{4} \geq \alpha, \quad r_{5} \geq\left(1-\frac{1}{1-\beta}\right) \alpha, \\
& \lambda_{i} \geq 0, \quad i=1, \ldots, 6, \\
& x \in \chi \text {, }
\end{aligned}
$$

where $x, \alpha, P_{i}, Q_{i}, p_{i}, q_{i}, r_{i}, s_{i}$, and $\lambda_{i}$ are variables. 
Proof. From $\min _{P \in \mathscr{P}} \mathbb{E}_{\xi}[-f(x, \xi)]=-\max _{P \in \mathscr{P}} \mathbb{E}_{\xi}[f(x, \xi)]$ and Theorem 4 in [19], we have that, for any given $x \in \mathbb{R}^{n}$, the problem $\min _{P \in \mathscr{P}} \mathbb{E}_{\xi}[-f(x, \xi)]$ can be transformed into the following problem:

$$
-\min \gamma_{2}\left(\bar{\Sigma} \cdot Q_{1}\right)-\bar{\mu}^{T} Q_{1} \bar{\mu}+r_{1}+\bar{\Sigma} \cdot P_{1}-2 \bar{\mu}^{T} p_{1}+\gamma_{1} s_{1}
$$

s.t. $\quad \xi^{T} Q_{1} \xi+\left(q_{1}+x\right)^{T} \xi+r_{1} \geq 0, \quad \forall \xi \in S$,

$$
\begin{aligned}
& \left(\begin{array}{cc}
P_{1} & p_{1} \\
p_{1}^{T} & s_{1}
\end{array}\right) \geq 0, \quad Q_{1} \geq 0, \\
& p_{1}=-\frac{q_{1}}{2}-Q_{1} \bar{\mu},
\end{aligned}
$$

where $P_{1}, Q_{1}, p_{1}, q_{1}, r_{1}$, and $s_{1}$ are variables.

Similarly to the above proof, for any given $x \in \mathbb{R}^{n}$, we have that $\max _{P \in \mathscr{P}} \min _{\alpha \in \mathbb{R}} F_{\beta}(x, \alpha)$ can be transformed into the following problem:

$$
\begin{aligned}
& \min \gamma_{2}\left(\bar{\Sigma} \cdot Q_{2}\right)-\bar{\mu}^{T} Q_{2} \bar{\mu}+r_{2}+\bar{\Sigma} \cdot P_{2}-2 \bar{\mu}^{T} p_{2}+\gamma_{1} s_{2} \\
& \text { s.t. } \quad \xi^{T} Q_{2} \xi+\xi^{T} q_{2}+r_{2} \geq \alpha, \quad \forall \xi \in S, \\
& \xi^{T} Q_{2} \xi+\xi^{T} q_{2}+r_{2} \geq \alpha-\frac{\alpha}{1-\beta}-\frac{x^{T} \xi}{1-\beta}, \quad \forall \xi \in S, \\
& \left(\begin{array}{cc}
P_{2} & p_{2} \\
p_{2}^{T} & s_{2}
\end{array}\right) \succeq 0, \quad Q_{2} \geq 0, \\
& p_{2}=-\frac{q_{2}}{2}-Q_{2} \bar{\mu},
\end{aligned}
$$

where $P_{2}, Q_{2}, p_{2}, q_{2}, r_{2}, s_{2}$, and $\alpha$ are variables. only if

In (8), $\xi^{T} Q_{1} \xi+\left(q_{1}+x\right)^{T} \xi+r_{1} \geq 0$ for any $\xi \in S$ if and

$$
\min _{\xi}\left\{\xi^{T} Q_{1} \xi+\left(q_{1}+x\right)^{T} \xi+r_{1}: \xi \in S\right\} \geq 0 .
$$

Because $Q_{1} \succeq 0$, the convex set $S$ has an interior point and objective function $\xi^{T} Q_{1} \xi+\left(q_{1}+x\right)^{T} \xi+r_{1}$ in the lefthand side of (10) is bounded below on $S$, and from the conic duality theorem, we know that (10) can be transformed into the following problem:

$$
\max _{r_{3}, \lambda_{1}, \lambda_{2}}\left\{\begin{array}{c}
\left.r_{3}: \begin{array}{cc}
r_{1}+\lambda_{1}^{T} \underline{\xi}-\lambda_{2}^{T} \bar{\xi}-r_{3} & \nu_{1}^{T} \\
\nu_{1}, \lambda_{2} \geq 0 & Q_{1}
\end{array}\right) \geq 0
\end{array}\right\} \geq 0,
$$

where $v_{1}=\left(q_{1}+x+\lambda_{2}-\lambda_{1}\right) / 2$, the left-hand side of (11), is dual problem of the left-hand side of (10) with the same optimal value. Similarly to the above proof, $\xi^{T} Q_{2} \xi+\xi^{T} q_{2}+r_{2} \geq \alpha$ for any $\xi \in S$ if and only if

$$
\max _{r_{4}, \lambda_{3}, \lambda_{4}}\left\{\begin{array}{c}
\left.r_{4}: \begin{array}{ll}
r_{2}+\lambda_{3}^{T} \underline{\xi}-\lambda_{4}^{T} \bar{\xi}-r_{4} & v_{2}^{T} \\
v_{2} & Q_{2}
\end{array}\right) \geq 0 \\
\lambda_{3}, \lambda_{4} \geq 0
\end{array}\right\} \geq \alpha,
$$

where $v_{2}=\left(q_{2}+\lambda_{4}-\lambda_{3}\right) / 2$. Similarly, $\xi^{T} Q_{2} \xi+\xi^{T} q_{2}+r_{2} \geq$ $(1-1 /(1-\beta)) \alpha-(1 /(1-\beta)) x^{T} \xi$ for any $\xi \in S$ if and only if

$$
\max _{r_{5}, \lambda_{5}, \lambda_{6}}\left\{\begin{array}{c}
\left.r_{5}: \begin{array}{ll}
\nu_{3} & \nu_{4}^{T} \\
\nu_{4} & Q_{2}
\end{array}\right) \geq 0 \\
\lambda_{5}, \lambda_{6} \geq 0
\end{array}\right\} \geq\left(1-\frac{1}{1-\beta}\right) \alpha
$$

where $\nu_{3}=r_{2}+\lambda_{5}^{T} \xi-\lambda_{6}^{T} \bar{\xi}-r_{5}$, and $\nu_{4}=\left(q_{2}+(1 /(1-\beta)) x+\right.$ $\left.\lambda_{6}-\lambda_{5}\right) / 2$.

From (8) and (11), we have that $\min _{P \in \mathscr{P}} \mathbb{E}_{\xi}[-f(x, \xi)]$ can be transformed into the following problem:

$$
\begin{array}{ll}
-\min & \gamma_{2}\left(\bar{\Sigma} \cdot Q_{1}\right)-\bar{\mu}^{T} Q_{1} \bar{\mu}+r_{1}+\bar{\Sigma} \cdot P_{1}-2 \bar{\mu}^{T} p_{1}+\gamma_{1} s_{1} \\
\text { s.t. } & \left(P_{1}, Q_{1}, p_{1}, q_{1}, r_{1}, r_{3}, s_{1}, \lambda_{1}, \lambda_{2}\right) \in \mathscr{C}_{1}(x),
\end{array}
$$

where

$\mathscr{C}_{1}(x)$

$$
=\left\{\begin{array}{c}
\left(P_{1}, \lambda_{1}, p_{1}, q_{1}, r_{1}, r_{3}, s_{1}, \lambda_{1}, \lambda_{2}\right):\left(\begin{array}{cc}
r_{2}-r_{3} & v_{1}^{T} \\
P_{1} & p_{1} \\
p_{1}^{T} & s_{1}
\end{array}\right) \geq 0, \quad Q_{1} \\
p_{1}=-\frac{q_{1}}{2}-Q_{1} \bar{\mu}, \quad r_{3}, \lambda_{1}, \lambda_{2} \geq 0
\end{array}\right\} .
$$

From (9), (12), and (13), we have that $\max _{P \in \mathscr{P}} \min _{\alpha \in \mathbb{R}} F_{\beta}(x, \alpha) \leq \sigma_{1}$ if and only if the set

$$
\left\{\begin{array}{c}
\bar{\Sigma} \cdot\left(\gamma_{2} Q_{2}+P_{2}\right)-\bar{\mu}^{T} Q_{2} \bar{\mu}+r_{2}-2 \bar{\mu}^{T} p_{2}+\gamma_{1} s_{2} \leq \sigma_{1} \\
\left(\begin{array}{cc}
r_{2}+\lambda_{3}^{T} \underline{\xi}-\lambda_{4}^{T} \bar{\xi}-r_{4} & \frac{\left(q_{2}+\lambda_{4}-\lambda_{3}\right)^{T}}{2} \\
\frac{\left(q_{2}+\lambda_{4}-\lambda_{3}\right)}{2} & Q_{2}
\end{array}\right) \geq 0 \\
\Theta:\left(\begin{array}{cc}
r_{2}+\lambda_{5}^{T} \underline{\xi}-\lambda_{6}^{T} \bar{\xi}-r_{5} & v_{4}^{T} \\
v_{4} & Q_{2}
\end{array}\right) \geq 0, \quad Q_{2} \geq 0 \\
\left(\begin{array}{cc}
P_{2} & p_{2} \\
p_{2}^{T} & s_{2}
\end{array}\right) \geq 0, \quad p_{2}=-\frac{q_{2}}{2}-Q_{2} \bar{\mu} \\
r_{4} \geq \alpha, \quad r_{5} \geq\left(\begin{array}{c}
1-\frac{1}{1-\beta}
\end{array}\right) \alpha, \quad \lambda_{3}, \lambda_{4}, \lambda_{5}, \lambda_{6} \geq 0
\end{array}\right\}
$$

(denoted by $\left.\mathscr{C}_{2}(x)\right)$ is nonempty, where $\Theta=\left(P_{2}, Q_{2}, p_{2}, q_{2}\right.$, $\left.s_{2}, r_{2}, r_{4}, r_{5}, \lambda_{3}, \lambda_{4}, \lambda_{5}, \lambda_{6}, \alpha\right)$.

From equality

$$
\max _{x} \min _{P \in \mathscr{P}} \mathbb{E}_{\xi}[-f(x, \xi)]=-\min _{x}\left\{-\min _{P \in \mathscr{P}} \mathbb{E}_{\xi}[-f(x, \xi)]\right\},
$$


(20), (15), and (16), we have that Model 1 can be restated equivalently as the following problem:

$$
\begin{array}{ll}
\max & -\left(\bar{\Sigma} \cdot\left(\gamma_{2} Q_{1}+P_{1}\right)-\bar{\mu}^{T} Q_{1} \bar{\mu}+r_{1}-2 \bar{\mu}^{T} p_{1}+\gamma_{1} s_{1}\right) \\
\text { s.t. } & \left(P_{1}, Q_{1}, p_{1}, q_{1}, r_{1}, r_{3}, s_{1}, \lambda_{1}, \lambda_{2}\right) \in \mathscr{C}_{1}(x), \\
& \left(P_{2}, Q_{2}, p_{2}, q_{2}, s_{2}, r_{2}, r_{4}, r_{5}, \lambda_{3}, \lambda_{4}, \lambda_{5}, \lambda_{6}, \alpha\right) \in \mathscr{C}_{2}(x), \\
& x \in \chi .
\end{array}
$$

Because the matrix inequality

$$
\left(\begin{array}{cc}
r_{1}+\lambda_{1}^{T} \underline{\xi}-\lambda_{2}^{T} \bar{\xi}-r_{3} & \frac{\left(q_{1}+x+\lambda_{2}-\lambda_{1}\right)^{T}}{2} \\
\frac{\left(q_{1}+x+\lambda_{2}-\lambda_{1}\right)}{2} & Q_{1}
\end{array}\right) \geq 0
$$

implies that $Q_{1} \geq 0$, the matrix inequality $Q_{1} \geq 0$ in $\mathscr{C}_{1}(x)$ is removed. Similarly, the matrix inequality $Q_{2} \geq 0$ in $\mathscr{C}_{2}(x)$ is removed. So our proof is complete.

Theorem 3. Model 2 can be reformulated as the following semidefinite programming problem:

$$
\begin{aligned}
& \min \bar{\Sigma} \cdot\left(\gamma_{2} Q_{2}+P_{2}\right)-\bar{\mu}^{T} Q_{2} \bar{\mu}+r_{2}-2 \bar{\mu}^{T} p_{2}+\gamma_{1} s_{2} \\
& \text { s.t. } \quad\left(\begin{array}{cc}
r_{1}+\lambda_{1}^{T} \underline{\xi}-\lambda_{2}^{T} \bar{\xi}-r_{3} & \frac{\left(q_{1}+x+\lambda_{2}-\lambda_{1}\right)^{T}}{2} \\
\frac{\left(q_{1}+x+\lambda_{2}-\lambda_{1}\right)}{2} & Q_{1}
\end{array}\right) \geq 0 \text {, } \\
& \left(\begin{array}{cc}
r_{2}+\lambda_{3}^{T} \underline{\xi}-\lambda_{4}^{T} \bar{\xi}-r_{4} & \frac{\left(q_{2}+\lambda_{4}-\lambda_{3}\right)^{T}}{2} \\
\frac{\left(q_{2}+\lambda_{4}-\lambda_{3}\right)}{2} & Q_{2}
\end{array}\right) \geq 0 \\
& \left(\begin{array}{cc}
r_{2}+\lambda_{5}^{T} \underline{\xi}-\lambda_{6}^{T} \bar{\xi}-r_{5} & \frac{\left(q_{2}+(x /(1-\beta))+\lambda_{6}-\lambda_{5}\right)^{T}}{2} \\
\frac{q_{2}+(x /(1-\beta))+\lambda_{6}-\lambda_{5}}{2} & Q_{2}
\end{array}\right) \geq 0, \\
& \left(\begin{array}{cc}
P_{1} & p_{1} \\
p_{1}^{T} & s_{1}
\end{array}\right) \succeq 0, \quad\left(\begin{array}{cc}
P_{2} & p_{2} \\
p_{2}^{T} & s_{2}
\end{array}\right) \succeq 0, \\
& -\left\{\gamma_{2}\left(\bar{\Sigma} \cdot Q_{1}\right)-\bar{\mu}^{T} Q_{1} \bar{\mu}+r_{1}+\bar{\Sigma} \cdot P_{1}-2 \bar{\mu}^{T} p_{1}+\gamma_{1} s_{1}\right\} \geq \sigma_{2}, \\
& p_{1}=-\frac{q_{1}}{2}-Q_{1} \bar{\mu}, \quad p_{2}=-\frac{q_{2}}{2}-Q_{2} \bar{\mu}, \\
& r_{3} \geq 0, \quad r_{4} \geq \alpha, \quad r_{5} \geq\left(1-\frac{1}{1-\beta}\right) \alpha, \\
& \lambda_{i} \geq 0, \quad i=1, \ldots, 6 \\
& x \in \chi
\end{aligned}
$$

where $x, \alpha, P_{i}, Q_{i}, p_{i}, q_{i}, r_{i}, s_{i}$, and $\lambda_{i}$ are variables.

Proof. The proof is similar to that of Theorem 2, so it is omitted.

Theorem 4. Model 3 can be reformulated as the following semidefinite programming problem:

$$
\begin{array}{ll}
\max & -\left(\gamma_{2}(\bar{\Sigma} \cdot Q)-\bar{\mu}^{T} Q \bar{\mu}+r_{1}+\bar{\Sigma} \cdot P-2 \bar{\mu}^{T} p+\gamma_{1} s\right) \\
\text { s.t. } & \left(\begin{array}{cc}
r_{1}+\lambda_{1}^{T} \xi-\lambda_{2}^{T} \bar{\xi}-r_{2} & \frac{\left(q+x+\lambda_{2}-\lambda_{1}\right)^{T}}{2} \\
\frac{\left(q+x+\lambda_{2}-\lambda_{1}\right)}{2} & Q
\end{array}\right) \geq 0,
\end{array}
$$$$
\left(\begin{array}{cc}
r_{1}+\lambda_{3}^{T} \underline{\xi}-\lambda_{4}^{T} \bar{\xi}-r_{3} & v_{5}^{T} \\
v_{5} & Q
\end{array}\right) \geq 0,
$$$$
\left(\begin{array}{cc}
P & p \\
p^{T} & s
\end{array}\right) \geq 0, \quad p=-\frac{q}{2}-Q \bar{\mu},
$$$$
r_{2} \geq \sigma_{3} \alpha, \quad r_{3} \geq\left(1-\frac{1}{1-\beta}\right) \alpha \sigma_{3},
$$$$
\lambda_{i} \geq 0, \quad i=1, \ldots, 4,
$$$$
x \in \chi
$$ 
where $\nu_{5}=\left(q+\left(1+\left(\sigma_{3} /(1-\beta)\right)\right) x+\lambda_{4}-\lambda_{3}\right) / 2$, and $x, \alpha, P, Q, p, q, r_{i}, s$, and $\lambda_{i}$ are variables.

Proof. From equality

$$
\begin{aligned}
& -\sigma_{3} \min _{\alpha \in \mathbb{R}} F_{\beta}(x, \alpha) \\
& =-\sigma_{3} \min _{\alpha \in \mathbb{R}} \int_{\xi \in \mathbb{R}^{m}}\left(\alpha+(1-\beta)^{-1}[f(x, \xi)-\alpha]^{+}\right) d P(\xi) \\
& =\max _{\alpha \in \mathbb{R}}\left[-\int_{\xi \in \mathbb{R}^{m}} \max \left(\alpha \sigma_{3}, \alpha \sigma_{3}+\frac{\sigma_{3}(f(x, \xi)-\alpha)}{1-\beta}\right) d P(\xi)\right] \\
& =\max _{\alpha \in \mathbb{R}} \int_{\xi \in \mathbb{R}^{m}} G_{1}(x, \xi) d P(\xi),
\end{aligned}
$$

where $G_{1}(x, \xi)=\min \left(-\alpha \sigma_{3},-\alpha \sigma_{3}-\left(\sigma_{3}(f(x, \xi)-\alpha) /(1-\beta)\right)\right)$, we have that

$$
\begin{aligned}
\mathbb{E}_{\xi} & {[-f(x, \xi)]-\sigma_{3} \min _{\alpha \in \mathbb{R}} F_{\beta}(x, \alpha) } \\
& =\max _{\alpha \in \mathbb{R}} \int_{\xi \in \mathbb{R}^{m}}\left(-f(x, \xi)+G_{1}(x, \xi)\right) d P(\xi) \\
& =\max _{\alpha \in \mathbb{R}} \int_{\xi \in \mathbb{R}^{m}} G_{2}(x, \xi) d P(\xi),
\end{aligned}
$$

where $G_{2}(x, \xi)=\min \left(-f-\alpha \sigma_{3},-f-\alpha \sigma_{3}-\left(\sigma_{3}(f-\alpha) /(1-\beta)\right)\right)$. Hence,

$$
\begin{aligned}
\min _{P \in \mathscr{P}}\left(\mathbb{E}_{\xi}[-f(x, \xi)]-\sigma_{3} \min _{\alpha \in \mathbb{R}} F_{\beta}(x, \alpha)\right) \\
=\min _{P \in \mathscr{P}} \max _{\alpha \in \mathbb{R}} \int_{\xi \in \mathbb{R}^{m}} G_{2}(x, \xi) d P(\xi) \\
=-\max _{P \in \mathscr{P}} \min _{\alpha \in \mathbb{R}} \int_{\xi \in \mathbb{R}^{m}}\left[-G_{2}(x, \xi)\right] d P(\xi) \\
=-\max _{P \in \mathscr{P}} \min _{\alpha \in \mathbb{R}} \int_{\xi \in \mathbb{R}^{m}} G_{3}(x, \xi) d P(\xi) \\
=-\min _{\alpha \in \mathbb{R}} \max _{P \in \mathscr{P}} \int_{\xi \in \mathbb{R}^{m}} G_{3}(x, \xi) d P(\xi),
\end{aligned}
$$

where $G_{3}(x, \xi)=\max \left(f+\alpha \sigma_{3}, f+\alpha \sigma_{3}+\left(\sigma_{3}(f-\alpha) /(1-\right.\right.$ $\beta))$ ); interchanging of the "min" and "max" operators in the last equality is obtained by using the minimax theorem ([22]). Hence,

$$
\begin{gathered}
\max _{x \in \chi} \min _{P \in \mathscr{P}}\left(\mathbb{E}_{\xi}[-f(x, \xi)]-\sigma_{3} \min _{\alpha \in \mathbb{R}} F_{\beta}(x, \alpha)\right) \\
=\max _{x \in \chi}\left(-\min _{\alpha \in \mathbb{R}} \max _{P \in \mathscr{P}} \int_{\xi \in \mathbb{R}^{m}} G_{3}(x, \xi) d P(\xi)\right) \\
=-\min _{x \in \chi} \min _{\alpha \in \mathbb{R}} \max _{P \in \mathscr{P}} \int_{\xi \in \mathbb{R}^{m}} G_{3}(x, \xi) d P(\xi) \\
=-\min _{x \in \chi, \alpha \in \mathbb{R}} \max _{P \in \mathscr{P}} \int_{\xi \in \mathbb{R}^{m}} G_{3}(x, \xi) d P(\xi) .
\end{gathered}
$$

Similarly to the proof of Theorem 2, we can prove that Model 3 can be reformulated as the problem (21). Therefore the proof of the theorem is concluded.

\section{Application in Portfolio Selection}

In this section, we apply the models discussed in the previous section to solve portfolio problem and compare the behavior of the solutions obtained by our models and the solutions obtained by the model in [23] and the model

$$
\begin{array}{ll}
\max _{x} & x^{T} \bar{\mu} \\
\text { s.t. } & x^{T} \bar{\Sigma} x \leq \sigma_{1}, \\
& x \in \chi,
\end{array}
$$

using nominal data. The equivalent semidefinite programming problems for our models are solved by interior point methods [24].

In the following application, decision variable $x$ means portfolio weights and random vector $\xi$ means daily logarithmic return of the stocks. For Example 2, we evaluated our portfolio optimization problem using a historical data set of four stocks (China Mingsheng Banking (600016), Sinopec Group (600028), Sany Heavy Machinery (600031), and China Unicom (600050)) over a horizon of 10 years (2003-2012), obtained from CSMAR Solution. The historical data set we choose consisted of daily closing price of each stock of Shanghai Stock Exchange. Daily logarithmic returns were calculated by daily closing price. The parameters $\bar{\mu}$ and $\bar{\Sigma}$ are assigned as the estimates of the mean and covariance matrix of daily logarithmic return.

Example 1 (see [23]). Let the mean and covariance matrix of daily return of seven stocks be as follows:

$$
\begin{aligned}
& \bar{\mu}=(0.11,0.11,0.10,0.09,0.09,0.12,0.09)^{T}, \\
& \bar{\Sigma}=\left(\begin{array}{ccccccc}
0.293 & -0.229 & -0.237 & -0.259 & 0.257 & -0.193 & 0.256 \\
-0.229 & 0.210 & 0.210 & 0.221 & -0.216 & 0.162 & -0.215 \\
-0.237 & 0.210 & 0.225 & 0.239 & -0.216 & 0.168 & -0.219 \\
-0.259 & 0.221 & 0.239 & 0.275 & -0.246 & 0.189 & -0.247 \\
0.257 & -0.216 & -0.216 & -0.246 & 0.256 & -0.185 & 0.254 \\
-0.193 & 0.162 & 0.168 & 0.189 & -0.185 & 0.142 & -0.188 \\
0.256 & -0.215 & -0.219 & -0.247 & 0.254 & -0.188 & 0.266
\end{array}\right) .
\end{aligned}
$$




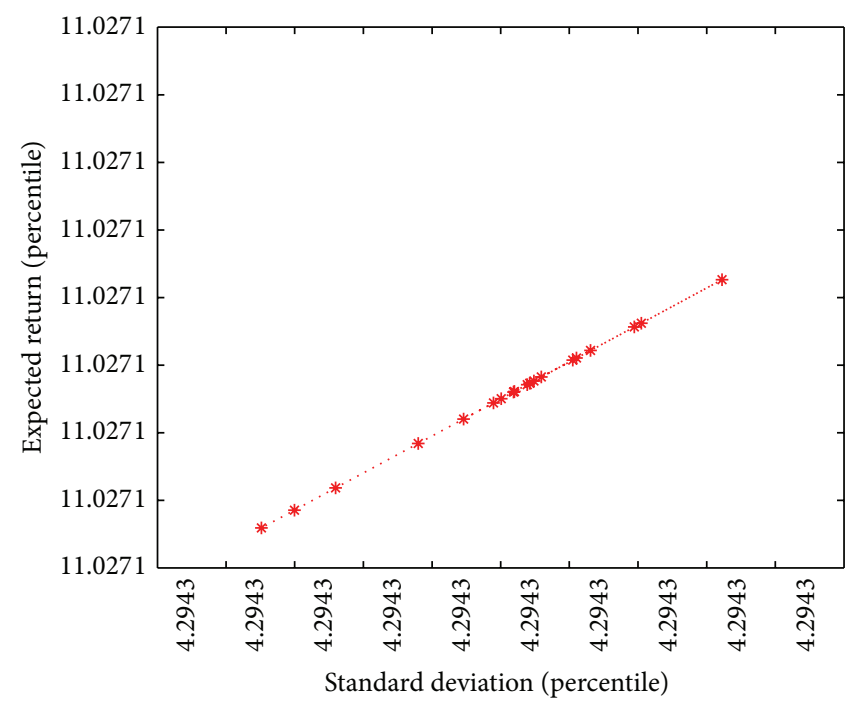

(a)

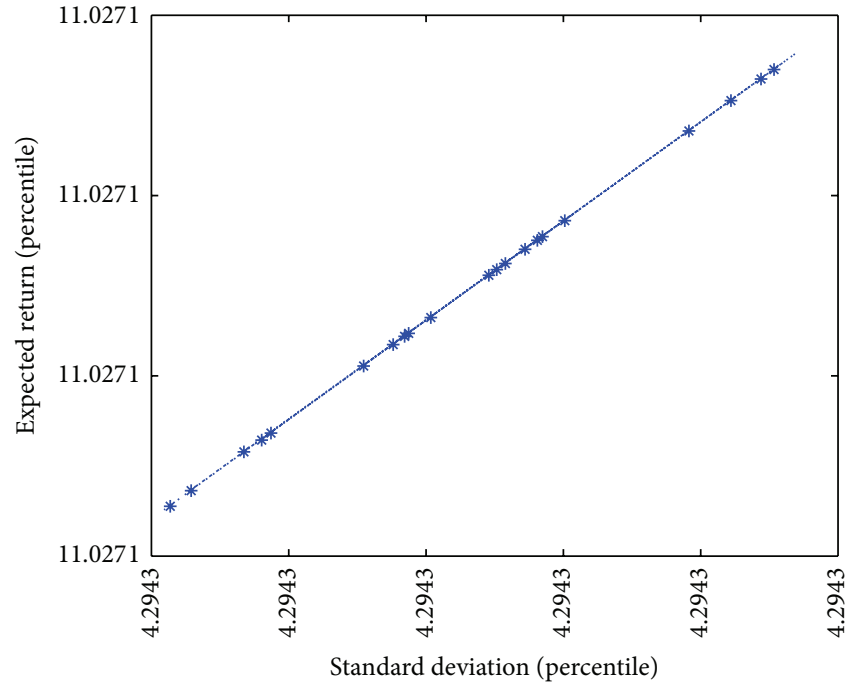

(b)

Figure 1: Efficient frontier obtained by Models 1 and 3 for Example 1.

TABLE 1: Numerical results for Example $1\left(\sigma_{1}=\sigma_{3}=0.2\right)$.

\begin{tabular}{lccc}
\hline Model & ER & SD & $($ WER, WCVaR) \\
\hline Model 1 & 0.1103 & 0.0429 & $(0.0868,0.2000)$ \\
Model 3 & 0.1103 & 0.0429 & 0.0468 \\
{$[21]$} & 0.1090 & 0.0417 & - \\
$(21)$ & 0.1200 & 0.3768 & - \\
\hline
\end{tabular}

TABle 2: Numerical results for Example $2\left(\sigma_{1}=\sigma_{3}=0.2\right)$.

\begin{tabular}{lccc}
\hline Model & ER & SD & $($ WER, WCVaR) \\
\hline Model 1 & $0.7161 e-04$ & 0.0209 & $(-0.0174,0.1976)$ \\
Model 3 & $0.6785 e-04$ & 0.0209 & -0.0400 \\
{$[21]$} & $0.6386 e-04$ & 0.0209 & - \\
$(21)$ & $0.2783 e-03$ & 0.0253 & - \\
\hline
\end{tabular}

Example 2. The empirical estimates $\bar{\mu}$ and $\bar{\Sigma}$ of the mean and covariance matrix of daily logarithmic return calculated by the historical data of the four stocks are as follows:

$$
\begin{aligned}
\bar{\mu} & =10^{-4}(-1.257,2.783,-3.273,0.276)^{T}, \\
\bar{\Sigma} & =\left(\begin{array}{llll}
0.0009 & 0.0003 & 0.0004 & 0.0003 \\
0.0003 & 0.0006 & 0.0003 & 0.0003 \\
0.0004 & 0.0003 & 0.0020 & 0.0003 \\
0.0003 & 0.0003 & 0.0003 & 0.0006
\end{array}\right) .
\end{aligned}
$$

The results for Examples 1 and 2 are listed in Tables 1 and 2, respectively. The tables give the expected return (ER) $\bar{\mu}^{T} x^{*}$, standard deviation (SD) $\sqrt{x^{* T} \bar{\Sigma} x^{*}}$, worst-case expected return, and worst-case CVaR ((WER,WCVaR), it is the risk-adjusted expected return function value (i.e., objective value) for Model 3 in tables), where $x^{*}$ is a global solution of models.
From tables and figures, we arrive at the following conclusion.

(1) We compare the models with the model in [23] and (26). The byproduct-solution pair $\left(\bar{\mu}^{T} x^{*}, \sqrt{x^{* T} \bar{\Sigma} x^{*}}\right)$, obtained by our models, is not worse than that obtained by the model in [23] and (26). Our model sometimes gives a slightly better result. That is, the investment decision with highest return is obtained. And our models give worst-case return and risk. So our model may be a better choice.

(2) Usually, the estimated mean and the covariance matrix of a return vector of the stocks based on the market data are subjected to errors. That is, there are errors between nominal data and true data. To analyze the robustness of the solution, for Example 1, let the nominal data be slightly perturbed. That is, let

$$
\begin{gathered}
\tilde{\mu}=\bar{\mu}, \\
\widetilde{\Sigma}=\bar{\Sigma}+\operatorname{diag}([0 ; 0 ; 0 ; 0.1 ; 0.1 ; 0.1 ; 0.1]),
\end{gathered}
$$

be the exact mean and covariance matrix, where "diag $(x)$ " denotes a diagonal matrix whose $i$ th diagonal element is $x_{i}$. Using the data to solve the models, the solution pair $\left(\bar{\mu}^{T} x^{*}, \sqrt{x^{* T} \bar{\Sigma} x^{*}}\right)$ obtained by model in [23] and (26) is $(0.1068,0.0611)$ and $(0.1200,0.3899)$, respectively. Hence, using results obtained by model in [23] and (26) in Table 1, investors tend to overestimate return and underestimate risk. From $\mathbb{E}_{\xi}[\xi]=\widetilde{\mu}=\bar{\mu}, \mathbb{E}_{\xi}[\xi-\widetilde{\mu}]\left(\mathbb{E}_{\xi}[\xi-\widetilde{\mu}]\right)^{T}=\widetilde{\Sigma}$, and $\widetilde{\Sigma} \preceq 5 \bar{\Sigma}$, we have that distribution of random vector $\xi$ with mean $\tilde{\mu}$ and covariance matrix $\widetilde{\Sigma}$ lies in the set $\mathscr{P}$. So our proposed methods are robust and the investment strategy is safe.

(3) By solving Models 1 and 3 for different values of $\sigma_{1}$ and $\sigma_{3}$, one can generate a sequence of optimal portfolios on the efficient frontier. From Figures 1 and 2, we observe that it ranges from the portfolio with the smallest overall variance to 


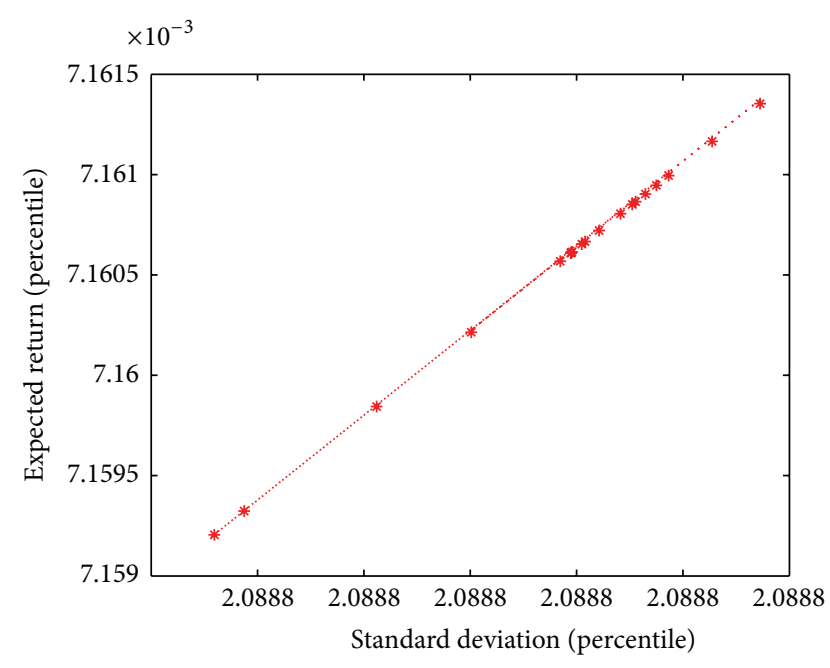

(a)

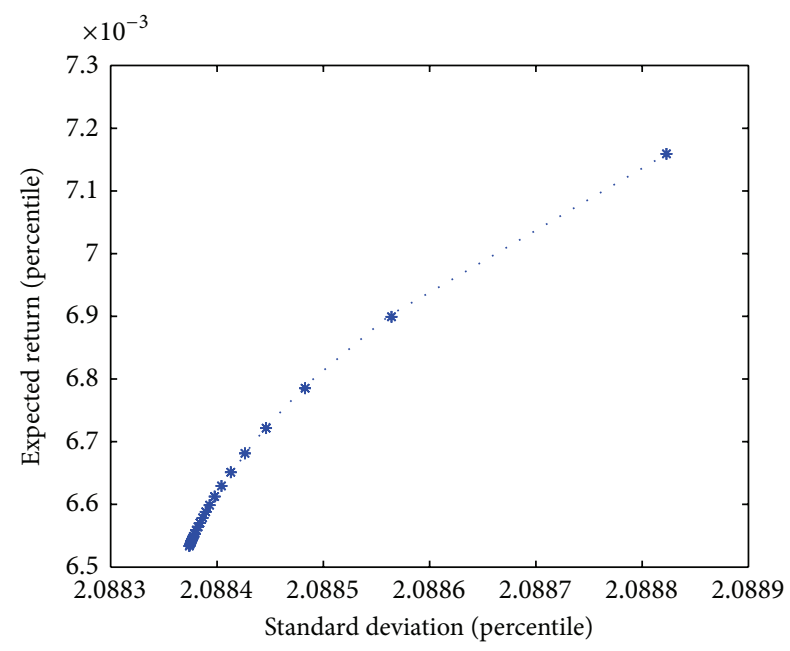

(b)

FIGURE 2: Efficient frontier obtained by Models 1 and 3 for Example 2.

the portfolio with the highest expected return; that is, efficient frontier is monotonically increasing. This is consistent with the reality.

\section{Conclusion}

We proposed distributionally robust return-risk optimization models based on the risk measure CVaR, with box constraints of random vector and uncertainty in both the distribution form and moments (mean and covariance matrix) of random vector. The models are reformulated as computationally tractable semidefinite programming problems. And the scale of semidefinite programming problems is independent of the number of sample points. Moreover, an application of the models to a practical example of portfolio selection with historical stock data shows that proposed methods are robust and the investment strategy obtained by them is safe. The models are suitable for investors with high security demands.

\section{Conflict of Interests}

The authors declare that they do not have any conflict of interests in their submitted paper.

\section{Acknowledgments}

The authors are grateful to anonymous reviewers for many helpful suggestions. Their suggestions led to an improved version of this paper. The work was supported by the National Natural Science Foundation of China (11301050, 11171051, 91230103, 71172136, and 71301017).

\section{References}

[1] H. Markowitz, "Portfolio selection," The Journal of Finance, vol. 7, no. 1, pp. 77-91, 1952.
[2] P. Artzner, F. Delbaen, J. M. Eber, and D. Heath, "Coherent measures of risk," Mathematical Finance, vol. 9, no. 3, pp. 203228, 1999.

[3] H. Mausser and D. Rosen, "Beyond VaR: from measuring risk to managing risk," ALGO Research Quarterly, vol. 1, no. 2, pp. 5-20, 1999.

[4] R. T. Rockafellar and S. Uryasev, "Optimization of conditional value-at-risk," Journal of Risk, vol. 2, no. 3, pp. 21-41, 2000.

[5] S. Uryasev and R. T. Rockafellar, "Conditional value-at-risk: optimization approach," in Stochastic Optimization: Algorithms and Applications, vol. 54 of Applied Optimization, pp. 411435, Kluwer Academic Publishers, Dordrecht, The Netherlands, 2001.

[6] X. Lin and Q. Gong, "Research on the efficient frontier of MeanCVaR under normal distribution condition," Management Sciences in China, vol. 17, no. 3, pp. 52-55, 2004.

[7] G. J. Alexander and A. M. Baptista, "A comparison of VaR and CVaR constraints on portfolio selection with the mean-variance model," Management Science, vol. 50, no. 9, pp. 1261-1273, 2004.

[8] F. Andersson, H. Mausser, D. Rosen, and S. Uryasev, "Credit risk optimization with conditional value-at-risk criterion," Mathematical Programming B, vol. 89, no. 2, pp. 273-291, 2001.

[9] C. Lim, H. D. Sherali, and S. Uryasev, "Portfolio optimization by minimizing conditional value-at-risk via nondifferentiable optimization," Computational Optimization and Applications, vol. 46, no. 3, pp. 391-415, 2010.

[10] W. Ogryczak and T. Śliwiński, "On solving the dual for portfolio selection by optimizing conditional value at risk," Computational Optimization and Applications, vol. 50, no. 3, pp. 591-595, 2011.

[11] A. Ben-Tal, L. El Ghaoui, and A. Nemirovski, Robust Optimization, Princeton University Press, Princeton, NJ, USA, 2009.

[12] D. Bertsimas, D. B. Brown, and C. Caramanis, "Theory and applications of robust optimization," SIAM Review, vol. 53, no. 3, pp. 464-501, 2011.

[13] W. Ogryczak, "Tail mean and related robust solution concepts," International Journal of Systems Science, vol. 45, no. 1, pp. 29-38, 2014. 
[14] J. Gao and N. Bian, "Portfolio selection based on the risk control of WCVaR," System Engineering: Theory \& Practice, vol. 29, no. 5, pp. 69-75, 2009.

[15] S. Zhu and M. Fukushima, "Worst-case conditional value-atrisk with application to robust portfolio management," Operations Research, vol. 57, no. 5, pp. 1155-1168, 2009.

[16] X. Tong and Q. Liu, "WCVaR risk analysis under the box discrete distribution and application," System Engineering: Theory \& Practice, vol. 30, no. 2, pp. 305-314, 2010.

[17] D. Bertsimas, X. V. Doan, K. Natarajan, and C. P. Teo, "Models for minimax stochastic linear optimization problems with risk aversion," Mathematics of Operations Research, vol. 35, no. 3, pp. 580-602, 2010.

[18] S. Zymler, D. Kuhn, and B. Rustem, "Distributionally robust joint chance constraints with second-order moment information," Mathematical Programming A, vol. 137, no. 1-2, pp. 167198, 2013.

[19] E. Delage and Y. Ye, "Distributionally robust optimization under moment uncertainty with application to data-driven problems," Operations Research, vol. 58, no. 3, pp. 595-612, 2010.

[20] R. Mansini, W. Ogryczak, and M. G. Speranza, “Twenty years of linear programming based portfolio optimization," European Journal of Operational Research, vol. 234, no. 2, pp. 518-535, 2014.

[21] W. F. Sharpe, “The Sharpe ratio," Journal of Portfolio Management, vol. 21, no. 1, pp. 49-58, 1994.

[22] A. Shapiro, "On duality theory of conic linear problems," in Semi-Infinite Programming, vol. 57 of Nonconvex Optimization and Its Applications, pp. 135-165, Kluwer Academic Publishers, Dordrecht, The Netherlands, 2001.

[23] X. Deng, "Decision on nonnegative investment proportional coefficient of portfolio for risk minimization," Pure and Applied Mathematics, vol. 23, no. 4, pp. 524-528, 2007.

[24] R. H. Tütüncü, K. C. Toh, and M. J. Todd, "Solving semidefinitequadratic-linear programs using SDPT3," Mathematical Programming B, vol. 95, no. 2, pp. 189-217, 2003. 


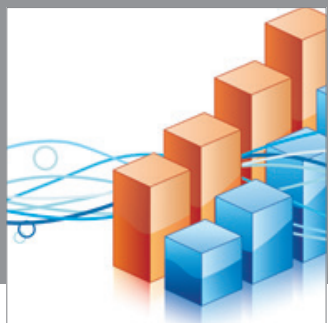

Advances in

Operations Research

mansans

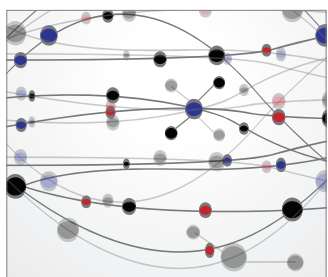

The Scientific World Journal
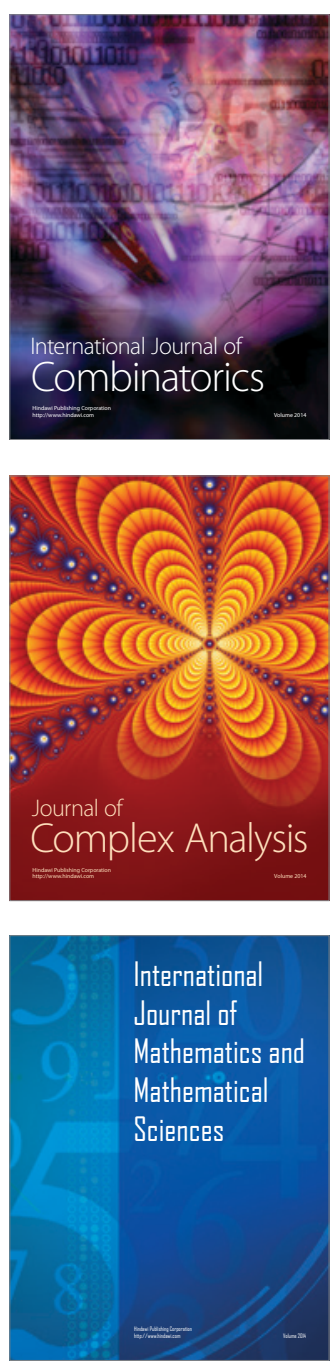
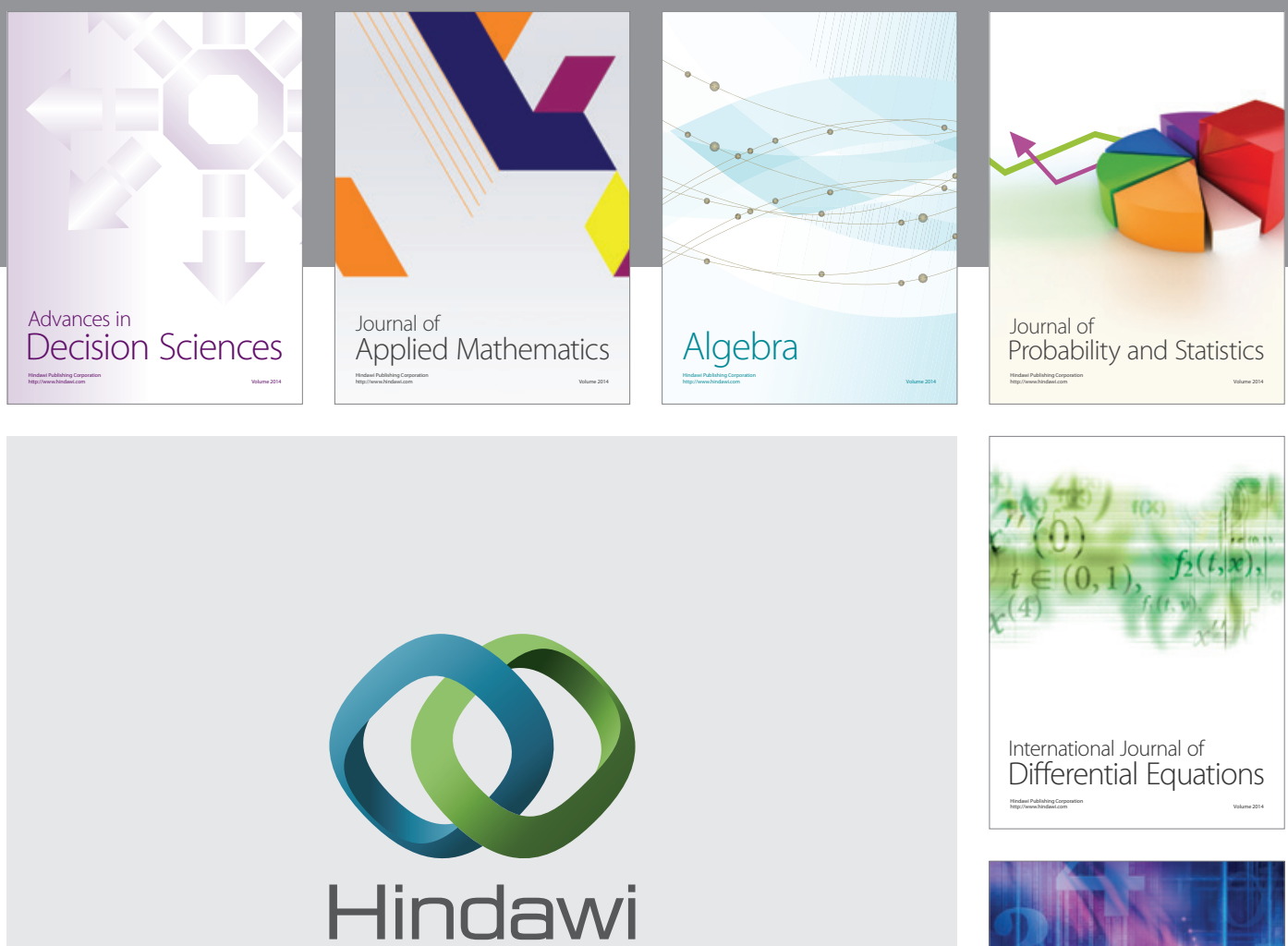

Submit your manuscripts at http://www.hindawi.com
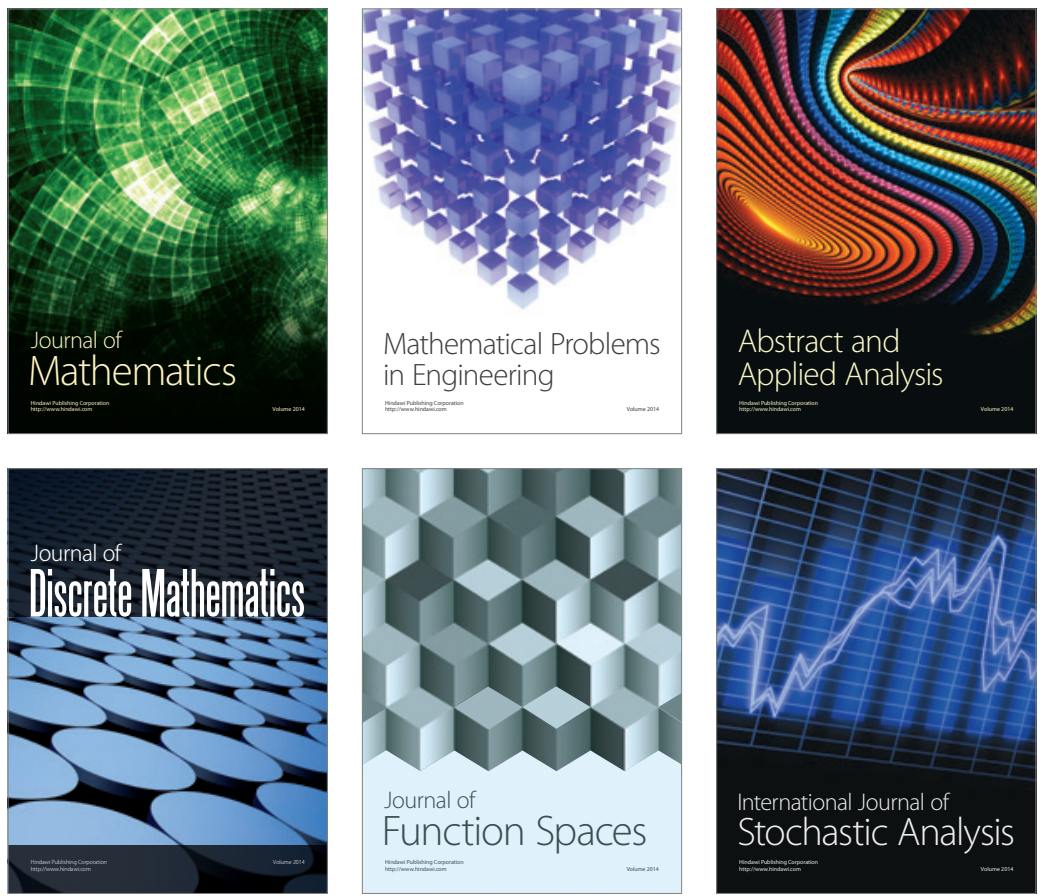

Journal of

Function Spaces

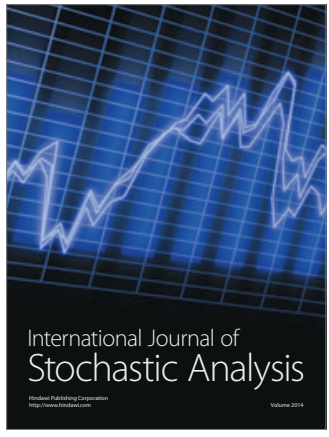

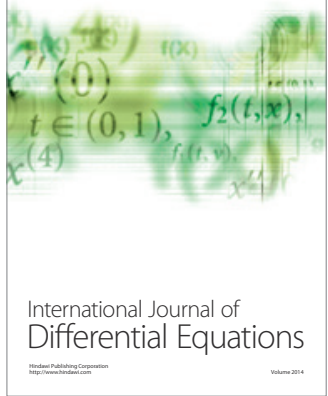
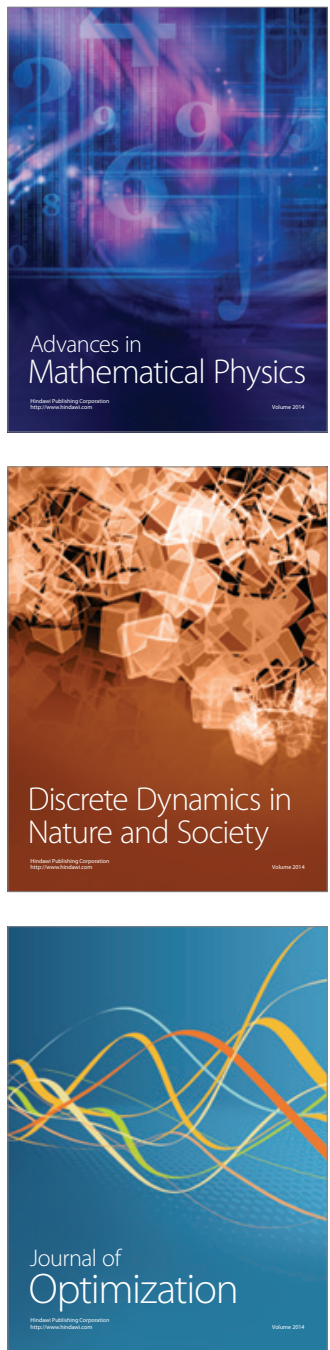